\title{
LITHIUM AND BERYLLIUM ISOTOPES IN THE PAMELA-EXPERIMENT
}

\author{
W. Menn ${ }^{* 19}$, O. Adriani ${ }^{5,6}$, G. C. Barbarino ${ }^{7,8}$, G. A. Bazilevskaya9 ${ }^{9}$, R. Bellotti $^{10,11}$, \\ M. Boezio ${ }^{2}$, E. A. Bogomolov ${ }^{12}$, M. Bongi ${ }^{4,6}$, V. Bonvicini ${ }^{2}$, S. Bottai ${ }^{6}$, A. Bruno ${ }^{10,11}$, \\ F. Cafagna ${ }^{11}$, D. Campana ${ }^{8}$, P. Carlson ${ }^{13}$, M. Casolino ${ }^{3,15}$, G. Castellini ${ }^{16}$, \\ C. De Donato ${ }^{3}$, C. De Santis ${ }^{14}$, N. De Simone ${ }^{3}$, V. di Felice ${ }^{3,4}$ V. Formato ${ }^{1,2}$, \\ A. M. Galper ${ }^{17}$, A. V. Karelin ${ }^{17}$, S. V. Koldashov ${ }^{17}$, S. Koldobskiy ${ }^{17}$, S. Y. Krutkov ${ }^{12}$, \\ A. N. Kvashnin ${ }^{9}$, A. Leonov ${ }^{17}$,V. Malakhov ${ }^{17}$, L. Marcelli ${ }^{14}$, M. Martucci ${ }^{14,18}$, \\ A. G. Mayorov ${ }^{17}$, M Merge ${ }^{3,14}$, V. V. Mikhailov $^{17}$,E. Mocchiutti ${ }^{2}$, A. Monaco ${ }^{10,11}$, \\ N. Mori ${ }^{6}$, R. Munini ${ }^{1,2}$, G. Osteria ${ }^{8}$, F. Palma ${ }^{3,14}$, B. Panico ${ }^{8}$ P. Papini ${ }^{6}$, M. Pearce $^{13}$, \\ P. Picozza ${ }^{3,14}$, M. Ricci ${ }^{18}$, S. B. Ricciarini ${ }^{16}$, R. Sarkar ${ }^{2}$, V. Scotti ${ }^{7,8}$, M. Simon ${ }^{19}$, \\ R. Sparvoli ${ }^{3,14}$, P. Spillantini ${ }^{5,6}$, Y. I. Stozhkov ${ }^{9}$, A. Vacchi $^{2}$, E. Vannuccini ${ }^{6}$, \\ G. Vasilyev ${ }^{12}$, S. A. Voronovi7, Y. T. Yurkin ${ }^{17}$, G. Zampa ${ }^{2}$, N. Zampa ${ }^{2}$, M. S. Potgieter \\ ${ }^{21}$, E.E. Vos ${ }^{21}$. \\ ${ }^{1}$ University of Trieste, Department of Physics, I-34147 Trieste, Italy \\ ${ }^{2}$ INFN, Sezione di Trieste I-34149 Trieste, Italy \\ 3 INFN, Sezione di Rome "Tor Vergata", I-00133 Rome, Italy \\ ${ }^{4}$ Agenzia Spaziale Italiana (ASI) Science Data Center, I-00044 Frascati, Italy \\ ${ }^{5}$ University of Florence, Department of Physics, I-50019 Sesto Fiorentino, Florence, Italy \\ ${ }^{6}$ INFN, Sezione di Florence, I-50019 Sesto Fiorentino, Florence, Italy \\ ${ }^{7}$ University of Naples "Federico II", Department of Physics, I-80126 Naples, Italy \\ ${ }^{8}$ INFN, Sezione di Naples, I-80126 Naples, Italy \\ ${ }^{9}$ Lebedev Physical Institute, RU-119991, Moscow, Russia \\ ${ }^{1} 0$ University of Bari, Department of Physics, I-70126 Bari, Italy \\ ${ }^{11}$ INFN, Sezione di Bari, I-70126 Bari, Italy \\ ${ }^{12}$ Ioffe Physical Technical Institute, RU-194021 St. Petersburg, Russia \\ ${ }^{13}$ KTH, Department of Physics, and the Oskar Klein Centre for Cosmoparticle Physics, AlbaNova \\ University Centre, SE-10691 Stockholm, Sweden \\ ${ }^{14}$ University of Rome "Tor Vergata", Department of Physics, I-00133 Rome, Italy \\ ${ }^{15}$ RIKEN, Advanced Science Institute, Wako-shi, Saitama, Japan \\ ${ }^{16}$ IFAC, I-50019 Sesto Fiorentino, Florence, Italy \\ ${ }^{17}$ National Research Nuclear University MEPhI, RU-115409 Moscow \\ ${ }^{18}$ INFN, Laboratori Nazionali di Frascati, Via Enrico Fermi 40, I-00044 Frascati, Italy \\ ${ }^{19}$ Universität Siegen, Department of Physics, D-57068 Siegen, Germany \\ ${ }^{20}$ INFN, Sezione di Perugia, I-06123 Perugia, Italy
}

E-mail: mennepamela.physik.uni-siegen.de 
On the 15th of June 2006, the PAMELA satellite-borne experiment was launched from the Baikonur cosmodrome and it has been collecting data since July 2006. The apparatus comprises a time-of-flight system, a silicon-microstrip magnetic spectrometer, a silicon-tungsten electromagnetic calorimeter, an anti-coincidence system, a shower tail counter scintillator and a neutron detector. The scientific objectives addressed by the mission are the measurement of the antiprotons and positrons spectra in cosmic rays, the hunt for antinuclei as well as the determination of light nuclei fluxes from hydrogen to oxygen in a wide energy range and with very high statistics. In this paper the identification capability for lithium and beryllium isotopes for two different techniques are presented, combining the rigidity measurement from the magnetic spectrometer with the velocity information derived either with the time-of-flight or with multiple $\mathrm{dE} / \mathrm{dx}$ measurements in the calorimeter. Preliminary results of the isotopic ratios will be presented.

The 34th International Cosmic Ray Conference,

30 July- 6 August, 2015

The Hague, The Netherlands

${ }^{*}$ Speaker. 


\section{Introduction}

Measurements of the isotopic composition of elements of the cosmic radiation provide significant constraints on cosmic ray source composition and cosmic ray transport and acceleration in the galaxy. The isotopes of $\mathrm{Li}, \mathrm{Be}$, and $\mathrm{B}$ in cosmic rays are pure spallation products of primary cosmic rays, mainly $\mathrm{C}, \mathrm{N}$, and $\mathrm{O}$, when they interact with interstellar matter during their propagation in the Galaxy. For example, the production of ${ }^{7} \mathrm{Li}$ in particular has important cosmological implications. While the ${ }^{6} \mathrm{Li}$ isotope is a pure product of interactions of galactic cosmic rays (GCR) with the interstellar medium (ISM), the ${ }^{7} \mathrm{Li}$ isotope has several sources. In addition to the production of ${ }^{7} \mathrm{Li}$ by spallation during the propagation of GCR, the stellar production and primordial nucleosynthesis of ${ }^{7} \mathrm{Li}$ are possible sources. All shown measurements (see Fig. 6 ) have values which are much smaller than the value $\sim 12$ determined from meteorites [䧃] or the value of $12.5 \pm 4.0$ if a stellar contribution to the interstellar medium is regarded [5].

A measurement of the isotopic composition of cosmic-ray beryllium is useful for determining the timescale for cosmic ray escape: ${ }^{7} \mathrm{Be}$ decays only by electron capture (half-life on earth $=53$ days), thus the half live in space depends on the electron density and the galactic cosmic ray lifetime. ${ }^{9} \mathrm{Be}$ is stable, but ${ }^{10} \mathrm{Be}$ has a half-life of $1.5 \times 10^{6}$ years years, which is comparable to the characteristic storage time expected for the galactic containment, and thus can be used to "date" the cosmic rays.

The PAMELA experiment has been observing galactic cosmic rays since July 2006 at an altitude ranging from $\sim 350 \mathrm{~km}$ to $\sim 600 \mathrm{~km}$ on-board of the Russian Resurs-DK1 satellite which executes a quasi-polar orbit $\left(70^{\circ}\right.$ inclination). The results presented here are based on the data set collected by PAMELA between July 2006 and September 2014.

\section{The PAMELA instrument}

The PAMELA satellite-borne cosmic ray experiment was built to measure charged particles in the cosmic radiation with a particular focus on antiparticles. It was mounted on the Resurs DK1 satellite and launched from the Baikonur cosmodrome on June 15th 2006. The PAMELA apparatus is composed of several sub-detectors: TOF system, anti-coincidence system (CARD, CAS, CAT), magnetic spectrometer with microstrip silicon tracking system, W/Si electromagnetic imaging calorimeter, shower-tail-catcher scintillator (S4) and neutron detector. A detailed description of the PAMELA instrument and an overview of the mission can be found in [6]. The core of the instrument is a magnetic spectrometer, made of a permanent magnet $(0.43 \mathrm{~T})$ and a silicon tracking system (resolution in the bending side $4 \mu \mathrm{m}$ ) for a maximum detectable rigidity of $1 \mathrm{TV}$. The Time of Flight (ToF) system is divided in 6 layers, arranged in three planes, each plane composed of two layers. The first plane (S1) is placed on top of the instrument, the second plane (S2) is placed between the TRD and the spectrometer, the last plane (S3) is placed between the spectrometer and the calorimeter, just below the magnet. The layers of S1 and S3 are $7 \mathrm{~mm}$ thick, while those of S2 are only $5 \mathrm{~mm}$ thick. The W/Si sampling imaging calorimeter comprises 44 single-sided silicon strip detector planes interleaved with 22 plates of tungsten absorber [ $[\mathbf{b}]$. Each tungsten layer has a thickness of 0.74 radiation lengths $(2.6 \mathrm{~mm})$ and it is sandwiched between two printed circuit boards, which house the silicon detectors as well as the frontend and digitizing electronics. Each silicon plane consists of $3 \times 3,380 \mu \mathrm{m}$ thick, $8 \times 8 \mathrm{~cm}^{2}$ detectors, segmented into 32 strips with a 
pitch of $2.4 \mathrm{~mm}$. The orientation of the strips for two consecutive silicon planes is shifted by 90 degrees, thus providing 2-dimensional spatial information. The total depth of the calorimeter is 16.3 radiation lengths and 0.6 nuclear interaction lengths.

\section{Data analysis}

\subsection{Event selection}

For this work we used the most recent reduction of the PAMELA flight data, collected between July 2006 and September 2014. In this reduction the tracking algorithm was modified, which resulted in a more precise fitting for light nuclei and a higher track finding efficiency. Also the time-walk correction for the ToF was improved, which lead to a better time resolution for light nuclei. The selection criteria applied to each triggered event will select positively charged particles with a precise measurement of the absolute value of the particle rigidity and velocity. Since we use events with rigidities only up to about $5 \mathrm{GV}$, our selection cuts are less strict compared to the analysis of lithium and beryllium spectra (up to some hundred $\mathrm{GeV} / \mathrm{n}$ ), which is presented

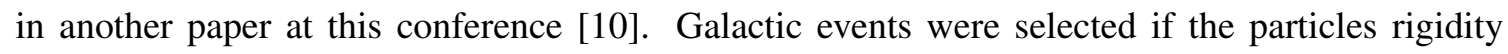
exceeded the critical rigidity, $\rho_{c}$, defined as 1.3 times the cutoff rigidity $\rho_{S V C}$ computed in the Störmer vertical approximation. Lithium and beryllium events have been selected by means of ionization energy losses in the TOF system. Charge consistency has been required between S12 and $<$ S2 $>$ and $<$ S3 $>$ (the arithmetic mean of the ionizations for the two layers constituting S2 and S3, respectively). Requiring charge consistency above and below the tracking system rejected events interacting in the silicon layers [B]].

\subsection{Isotope separation in the PAMELA instrument}

In each sample of $Z=3$ and $Z=4$ particles an isotopic separation at fixed rigidity is possible since the mass of each particle follows the relation $m=R Z e / \gamma \beta c$ ( $R$ is the magnetic rigidity, $Z \times e$ is the particle charge, and $\gamma$ is the Lorentz factor). The particle velocity $\beta$ can either be provided directly from the timing measurement of the ToF system, or indirectly from the energy loss in the calorimeter, which follows $\beta$ via the Bethe-Bloch formula $d E / d x \propto \frac{Z^{2}}{\beta^{2}}$ (neglecting logarithmic terms).

\subsubsection{Isotope separation using ToF vs. rigidity}

For the ToF analysis we can use directly the $\beta$ provided by the timing measurement. In Fig. $\square$ we show $\beta$ vs. the particle rigidity for $Z=3$ and $Z=4$ data. The black lines in the figure represent the expectations for each isotope.

\subsubsection{Isotope separation using multiple $d E / d x$ in the calorimeter vs. rigidity}

The isotopic analysis of nuclei with the calorimeter is restricted to events which do not interact inside the calorimeter, selecting events by applying cuts on the ratio between the energy deposited in the strip closest to the track and the neighboring strip on each side and the total energy detected. In a single silicon layer, the energy loss distributions shows a Landau tail which degrades the resolution of the $\mathrm{dE} / \mathrm{dx}$ measurement. Using a truncation method, the $50 \%$ of samples with larger 

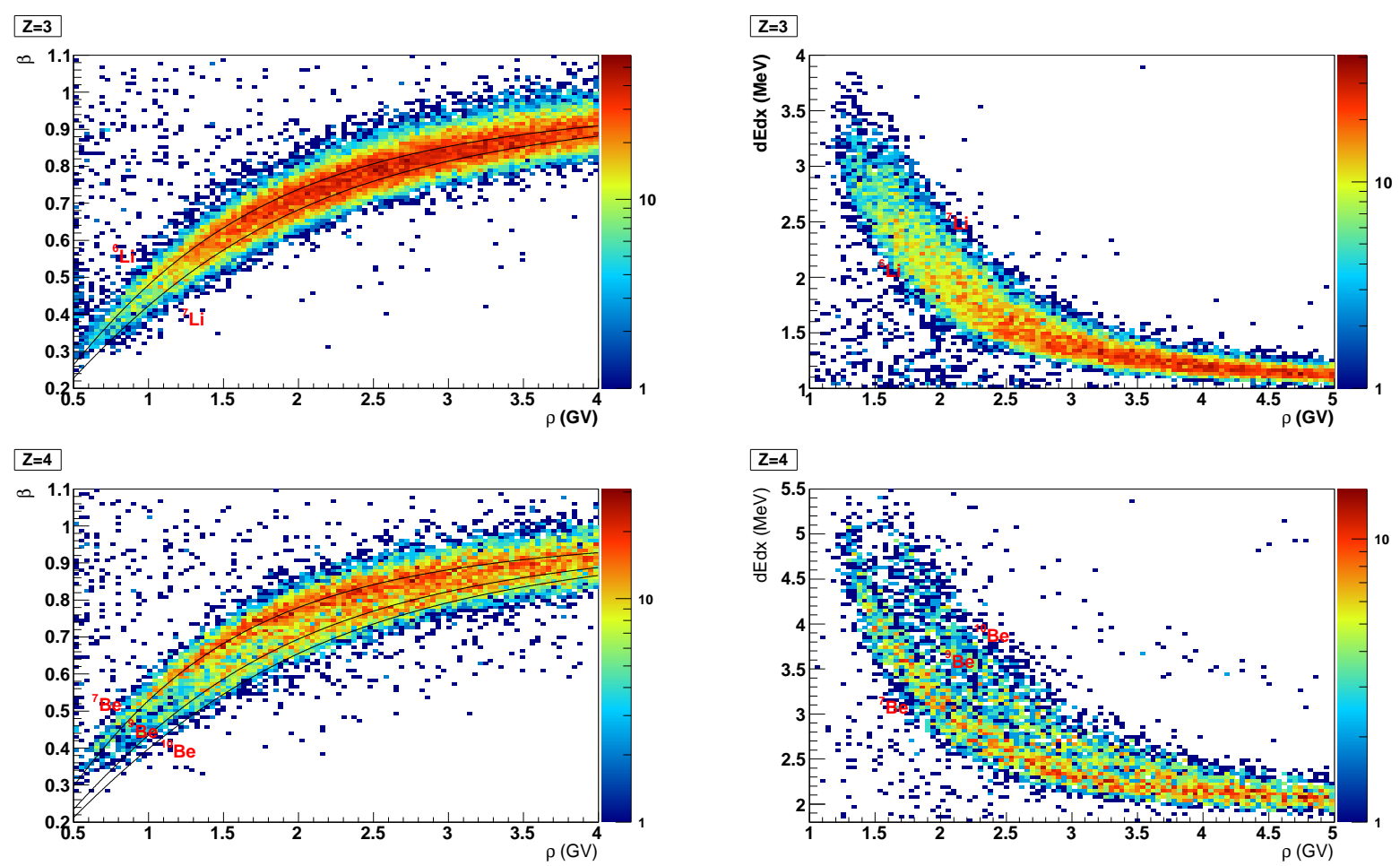

Figure 1: $\beta$ vs. rigidity for $Z=3$ (top) and $Z=4$ Figure 2: Mass separation for $Z=3$ (top) and $Z=4$ (bottom) particles. The black lines represent the expectations for each isotope.

pulse amplitudes are excluded before taking the mean of the $\mathrm{dE} / \mathrm{dx}$ measurements, thus reducing the effect of the Landau tail. The principle is described in more detail in another paper of this conference [Q]. In Fig. $\square$ the mean $\mathrm{dE} / \mathrm{dx}$ for each event vs. the rigidity for $Z=3$ and $Z=4$ particles is shown. The energy loss in MeV was derived from the measurement in MIP using a conversion factor. In both plots the isotopic separation is clearly visible. Note that the improved truncation method has a higher efficiency compared to the selection presented in earlier conferences []] ].

\subsubsection{Measured Mass resolution and comparison with expectations}

Theoretically, there are three independent contributions to the mass resolution in a magnet spectrometer similar to PAMELA: the precision of the velocity measurement (given either by timing or by measuring the energy loss), the bending power of the magnetic spectrometer coupled with the intrinsic limits of spatial resolution which the tracking detectors provide $\left(M D R_{\text {spec }}\right)$, and the multiple scattering of the particle along its path in the bending area of the magnet $\left(M D R_{\text {cou }}\right)$. The overall momentum resolution of the PAMELA magnetic spectrometer has been measured in beam tests at CERN ([目]): From the measurements at high energies, where the contributions from multiple scattering is negligible, one can derive that the $M D R_{\text {spec }}$ of the PAMELA spectrometer has a value of about $1 \mathrm{TV}$ for $Z=1$ particles. For light nuclei like $Z=3$ and $Z=4$ particles the tracking algorithm was modified, which resulted in a more precise fitting and a higher track finding 
efficiency, but resulting in an increased spatial resolution [四], which will reduce the $M D R_{\text {spec }}$ to about $500 \mathrm{GV}$. In any case the contribution of $M D R_{\text {spec }}$ to the overall mass resolution is negligible for the particles we are analyzing (up to some GV), at low energies the contribution from multiple scattering is however the dominant effect. Its value is inverse proportional to the bending power of the magnet $\left(\int B \cdot d l\right)$ and direct proportional to the amount of matter traversed along the bending part of the track. PAMELA combines a strong magnetic field of $0.45 \mathrm{~T}$ with a low amount of material (only the six silicon detectors, each $300 \mu \mathrm{m}$, giving a grammage of $0.42 \mathrm{~g} / \mathrm{cm}^{2}$ ) in the magnetic cavity, thus giving a respective value for $M D R_{\text {сои }}$ around $3.5 \%$ at $8 \mathrm{GV}$, increasing to about $5 \%$ at $1 \mathrm{GV}$ [目]. In Fig. B] the three independent contributions to the mass resolution for ${ }^{6} \mathrm{Li}$ and ${ }^{7} \mathrm{Be}$ particle from rigidity $\left(M D R_{\text {spec }}\right)$, multiple scatter $\left(M D R_{\text {cou }}\right)$, and velocity via ToF (time resolution $85 \mathrm{ps}$ for lithium and $80 \mathrm{ps}$ for beryllium nuclei) are shown as dotted lines. The time resolution of the ToF was derived from flight data using the width of $1 / \beta$ distributions at high rigidities. The expected mass resolution for the multiple $d E / d x$ versus rigidity technique was obtained using the GEANT 4 simulation. The simulated data was also used to parametrize the truncated mean value (in $\mathrm{MeV}$ ) as a function of the rigidity for each isotope, which was then used to convert from $\mathrm{MeV}$ to mass (in amu) using interpolation between the functions.

To derive the mass resolution for flight data we selected a sample of ${ }^{6} \mathrm{Li}$ or ${ }^{7} \mathrm{Be}$ particles using strict cuts on the mass coming from the ToF or multiple $d E / d x$. Then one can test the mass resolution of the ToF for the sample derived with multiple $d E / d x$ and vice versa (assuming that the contamination from other isotopes is negligible). As one can see the experimental results on the mass resolution follow nicely the prediction. This gives us confidence that we understand our instrument. It can also be seen that the multiple $d E / d x$ from the calorimeter provide a better mass resolution at higher energies than measuring the velocity via the ToF. This allows us to extend the PAMELA measurements on isotopes to higher energies. It can also be clearly seen that the multiple scatter sets the lower limit of the mass resolution at rigidities below roughly $2 \mathrm{GV}$ no matter how high the MDR of the spectrometer is. It should be noted that the mass resolution using the multiple $d E / d x$ from the calorimeter can be improved by using a more advanced analysis than the simple truncated mean. For example we calculated the energy loss in each calorimeter layer using the Bethe-Bloch equation, and then compared it with the data by leaving the mass of the particle as a free parameter [ $[8]$. However, these alternative approaches are still under development, for this work the simple truncated mean was used.

\subsection{Raw isotope numbers}

We had two complementing experimental methods to separate the isotopes: The combination of magnetic spectrometer either with the TOF or with the multiple $d E / d x$ measurements within the calorimeter. The isotope separation as well as the determination of isotope ratios was performed identical to [四] in intervals of kinetic energy per nucleon. Since the magnetic spectrometer measures the rigidity of particles, this implies different rigidity intervals according to mass of the the isotope under study.

\subsubsection{Raw isotope numbers with the ToF and calorimeter}

The $d E / d x$ distributions of the calorimeter have a non-gaussian shape, therefore one has to model the expected distributions of the observable quantities and then perform likelihood fits, we 

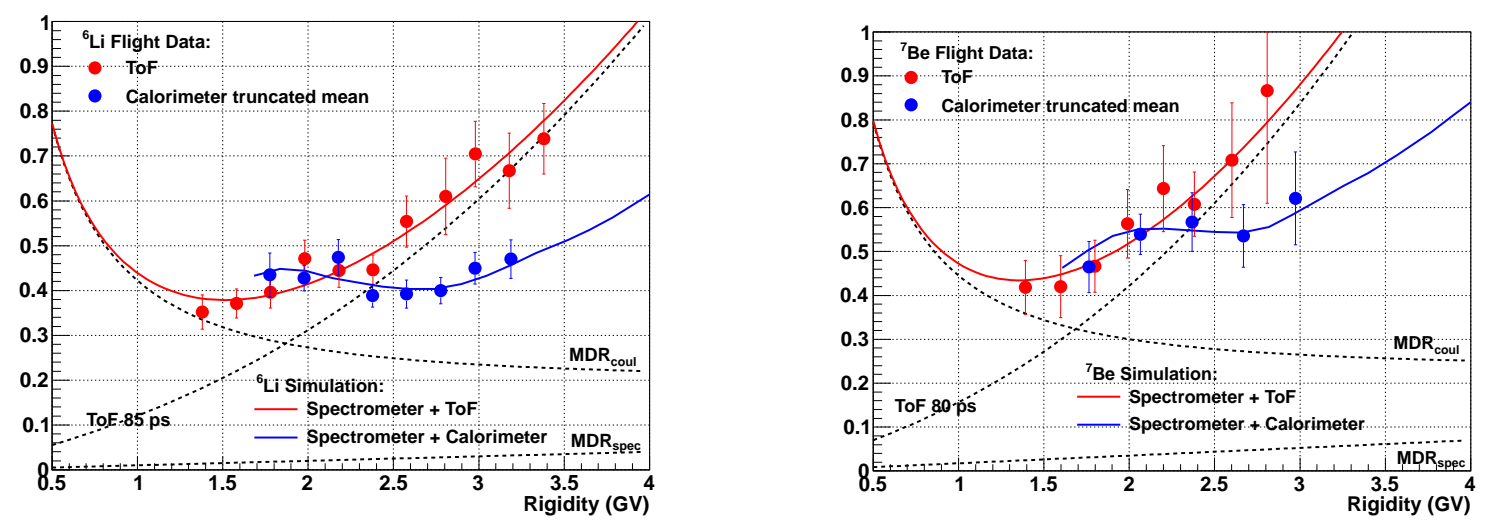

Figure 3: Expected and measured mass resolution for ${ }^{6} \mathrm{Li}$ particles(left) and ${ }^{7} \mathrm{Be}$ particles (right)

used the "TFractionFitter" toolkit for this task. First one has to create the expected $d E / d x$ distributions for each isotope, this is done using the full Monte Carlo simulation of the PAMELA apparatus based on the GEANT 4 code, which has been already described [8], [G], [四]. When analyzing hydrogen and helium isotopes, taking the simulated energy loss in each layer as coming from GEANT 4, we noticed that the resulting distributions showed a slight mismatch from the flight data. We found that the width of the histograms was smaller than in the real data, also there was a small offset of about $1-2 \%$. We applied a multiplicative factor to the simulated energy loss in a layer, plus adding a gaussian spread of the signal of some percent.

The $1 / \beta$ distributions of the ToF have a gaussian shape and could be fitted with gaussian functions, as it is done in the analysis of the hydrogen and helium isotopes ([四] [可]. However, in this work we used the same approach as for the calorimeter and created $1 / \beta$ distributions using the simulation, and then used the "TFractionFitter" toolkit for the Likelihood analysis.

As an example we show in Fig. $\mathbb{\text { ⿴囗十 }}$ the distributions ToF and calorimeter for lithium in the 1.9 $2.1 \mathrm{GV}$ rigidity range, and in Fig. $[$ similar distributions for beryllium. The grey area shows how the combined fit using the two (for lithium) or three (for beryllium) distributions derived with the modified GEANT 4 simulation matches the data points (black points) while the colored areas shows the estimated individual isotope signals.

\section{Isotopic ratios}

To derive the isotopic ratios, the numbers of selected events derived in the previous section had to be corrected for the selections efficiencies and particle losses. The efficiencies were mostly derived using simulations, these results were then checked using flight data at low energies, where the creation of test samples is possible using redundant detectors. Furthermore one needs the geometrical factor and the live time of the instrument as evaluated by the trigger system [四, since these values depend on the rigidity. The finite resolution of the magnetic spectrometer and particle slowdown due to ionization energy losses results in a distortion of the particle spectra, which affects the isotopic ratio. Work is in progress to employ a Bayesian unfolding procedure to correct for this effect ([山], [四], [Q] ]). 

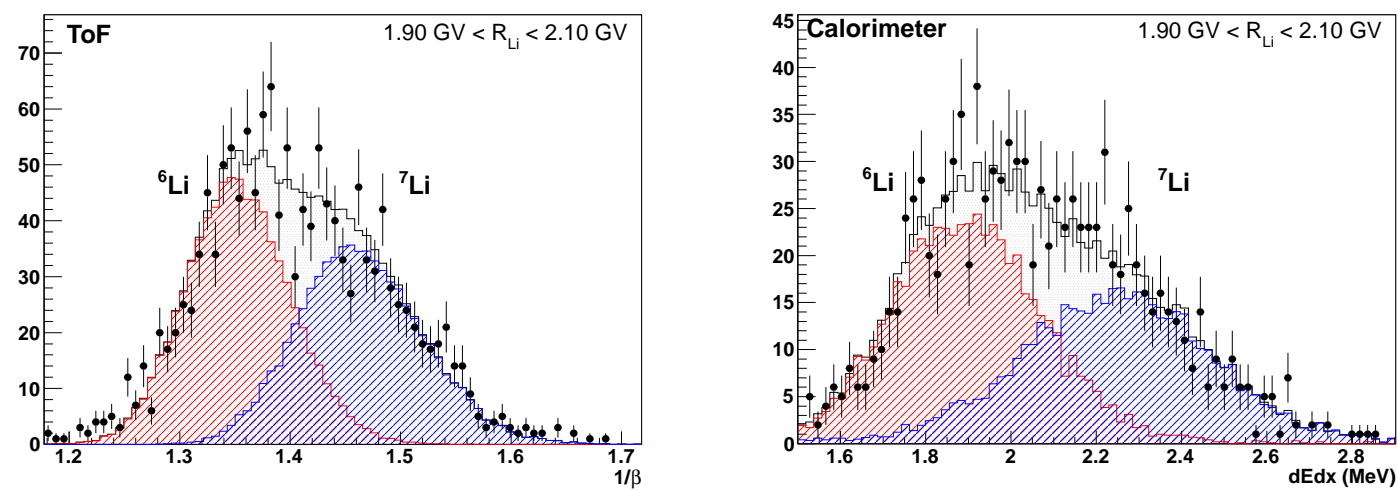

Figure 4: $1 / \beta$ distributions of the ToF (left) and truncated mean $d E / d x$ of the calorimeter (right) for lithium in the $1.9-2.1 \mathrm{GV}$ range.
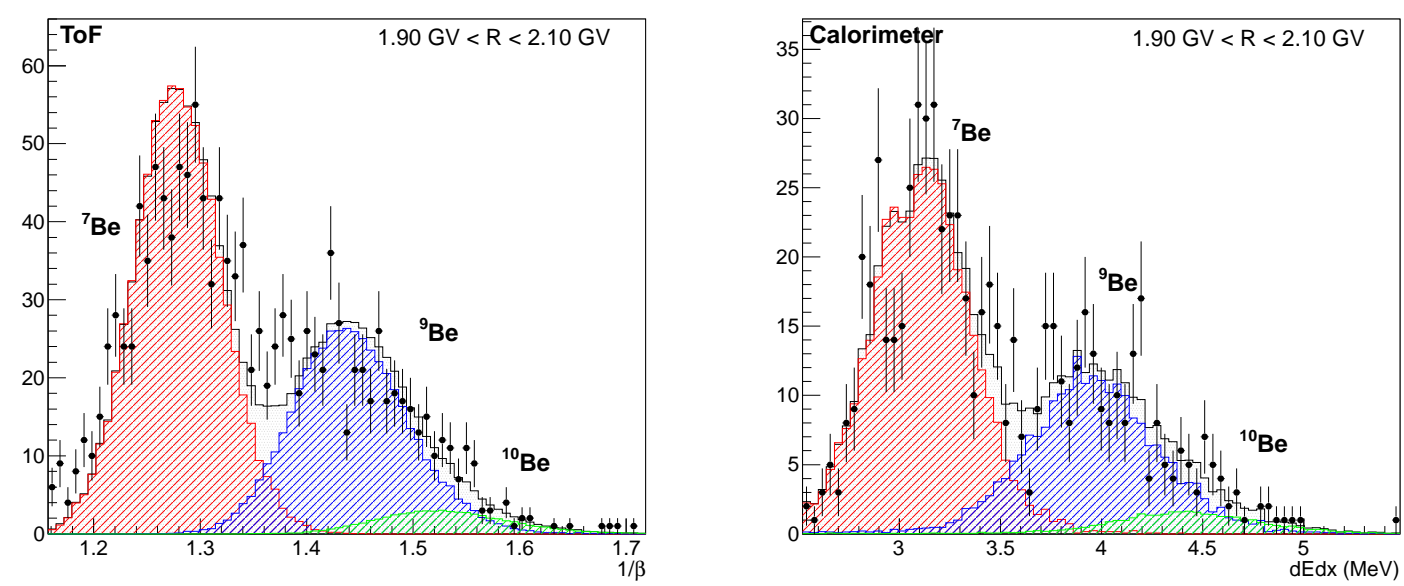

Figure 5: $1 / \beta$ distributions of the ToF (left) and truncated mean $d E / d x$ of the calorimeter (right) for beryllium in the $1.9-2.1 \mathrm{GV}$ range.

\section{Results and discussion}

In the following we show some preliminary results for the ratios ${ }^{7} \mathrm{Li} /{ }^{6} \mathrm{Li}$ and ${ }^{7} \mathrm{Be} /\left({ }^{9} \mathrm{Be}+{ }^{10} \mathrm{Be}\right)$ derived with the ToF (blue circles) or the calorimeter (red circles) together with other measurements. These preliminary results show a good agreement between the measurements with the ToF and the calorimeter, which gives confidence in the results. Work is in progress, especially the systematic error of the ratios will be studied in detail.

\section{References}

[1] O. Adriani et al. 2013 Astrophys. J. 7702

[2] O. Adriani et al. 2011, Science 33269 - Supplementary Online Material

[3] O. Adriani et al. 2014 Astrophys. J. 79130 

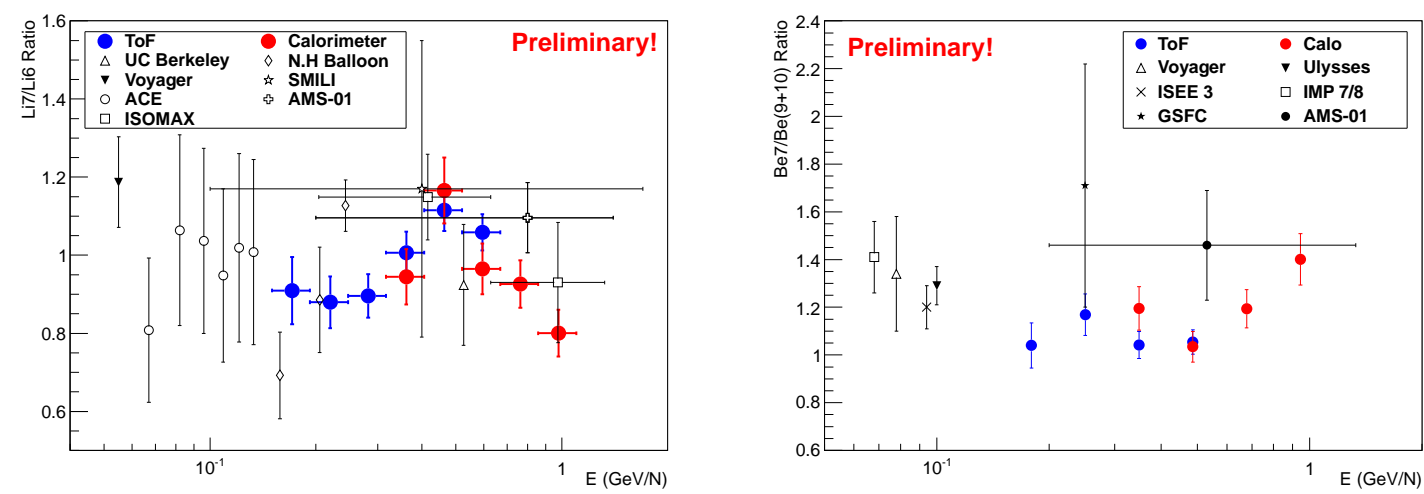

Figure 6: Results for the ratios ${ }^{7} \mathrm{Li} /{ }^{6} \mathrm{Li}$ and ${ }^{7} \mathrm{Be} /\left({ }^{9} \mathrm{Be}+{ }^{10} \mathrm{Be}\right)$ derived with the ToF (blue circles) or the calorimeter (red circles). Error bars show statistical uncertainty only. Other experiments: AMS-01 [ए2],

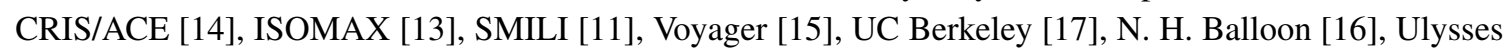

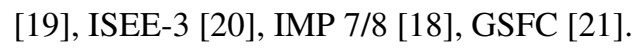

[4] E. Anders and N. Grevesse 1989 Geochim. Cosmochim. Acta 53197

[5] M. Lemoine et al. 1993 Astron. Astrophys. 269469

[6] P. Picozza et al. 2007 Astropart. Phys. 27 296-315

[7] M. Boezio et al. 2002 Nucl. Instr. and Meth. A 487 407-422

[8] W. Menn et al. 2013 Proc. 33nd Int. Cosmic Ray Conf. (Rio de Janeiro) 0233

[9] W. Menn et al. 2015 "New measurement of the isotopic composition of hydrogen and helium nuclei in cosmic rays with the PAMELA experiment"', this conference

[10] E. Vannuccini et al. 2015 "Measurement of Lithium and Beryllium cosmic-ray abundances by the PAMELA experiment"', this conference

[11] S. P. Ahlen et al. 2000 Astrophys. J. 534757

[12] M. Aguilar et al. 2011 Astrophys. J. 736105

[13] T. Hams et al. 2004 Astrophys. J. 611 892-905

[14] G. A. de Nolfo et al. 2006 Adv. Space Res. 381558 âĂŞ1564

[15] W. R. Webber, A. Lukasiak and F. B. McDonald 2002 Astrophys. J. 568 210-215

[16] W. R. Webber and J. Kish 1979 Proc. 16th Int. Cosmic Ray Conf. (Kyoto) 1389

[17] A. Buffington, C. D. Orth and T. S. Mast 1978 Astrophys. J. 226355

[18] M. Garcia-Munoz, G. M. Mason and J. A. Simpson 1977 Astrophys. J 217859

[19] J. J. Connell 1998 Astrophys. J. 501 L59

[20] M. E. Wiedenbeck and D. E. Greiner 1980 Astrophys. J. 239 L139

[21] F. A. Hagen, A. J. Fisher, and J. F. Ormes 1977 Astrophys. J. 212262 